\title{
Hybrid III-V/SOI single-mode vertical-cavity laser with in-plane emission into a silicon waveguide
}

Park, Gyeong Cheol; Xue, Weiqi; Semenova, Elizaveta; Mørk, Jesper; Chung, II-Sug

Published in:

Proceedings of 2015 Conference on Lasers and Electro-Optics (CLEO)

Link to article, DOI:

10.1364/CLEO_SI.2015.SW3F.2

Publication date:

2015

Document Version

Peer reviewed version

Link back to DTU Orbit

Citation (APA):

Park, G. C., Xue, W., Semenova, E., Mørk, J., \& Chung, I-S. (2015). Hybrid III-V/SOI single-mode vertical-cavity laser with in-plane emission into a silicon waveguide. In Proceedings of 2015 Conference on Lasers and ElectroOptics (CLEO) IEEE. https://doi.org/10.1364/CLEO_SI.2015.SW3F.2

\section{General rights}

Copyright and moral rights for the publications made accessible in the public portal are retained by the authors and/or other copyright owners and it is a condition of accessing publications that users recognise and abide by the legal requirements associated with these rights.

- Users may download and print one copy of any publication from the public portal for the purpose of private study or research.

- You may not further distribute the material or use it for any profit-making activity or commercial gain

- You may freely distribute the URL identifying the publication in the public portal 


\title{
Hybrid III-V/SOI single-mode vertical-cavity laser with in-plane emission into a silicon waveguide
}

\author{
Gyeong Cheol Park, Weiqi Xue, Elizaveta Semenova, Jesper Mørk, and Il-Sug Chung* \\ Department of Photonics Engineering, Technical University of Denmark, DK-2800 Kgs. Lyngby, Denmark \\ *ilch@fotonik.dtu.dk
}

\begin{abstract}
We report a III-V-on-SOI vertical-cavity laser emitting into an in-plane Si waveguide fabricated by using CMOS-compatible processes. The fabricated laser operates at $1.54 \mu \mathrm{m}$ with a SMSR of $33 \mathrm{~dB}$ and a low threshold.

(C) 2014 Optical Society of America

OCIS codes: (130.3120) Integrated optics devices; (140.2020) Diode lasers; (200.4650) Optical Interconnects.
\end{abstract}

\section{Introduction}

Vertical-cavity surface-emitting lasers (VCSELs) are preferred sources for short-reach optical communication applications due to their small energy consumption, high direct modulation speed, and efficient vertical light coupling to an optical fiber. Despite of these attractive properties, the conventional VCSEL is not applicable as light source for chip-level optical interconnects due to the difficulty of coupling the light output into an in-plane silicon (Si) waveguide. Recently, we have proposed the hybrid vertical cavity laser (hVCL) structure that can emit light directly into an in-plane Si waveguide [1]. This novel laser structure inherits all the excellent properties of conventional VCSELs, and may even further increase the speed since it has a much shorter photon lifetime as well as a higher confinement factor thanks to the high-index-contrast grating (HCG) reflector employed in the laser structure. Thus, the hVCL appears to be an attractive low-cost high-performance light source for the chip-level optical interconnects. In this paper, we report experimental results on the single-mode and low-threshold characteristics of the hVCL.

(a)

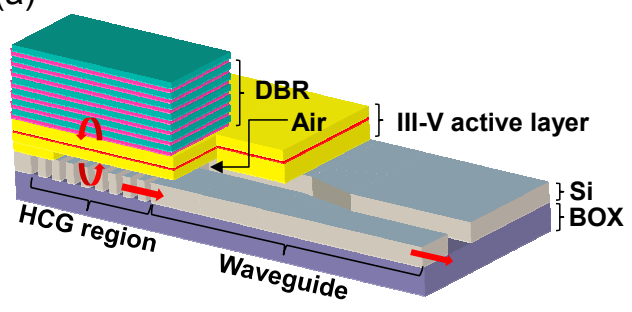

(b)

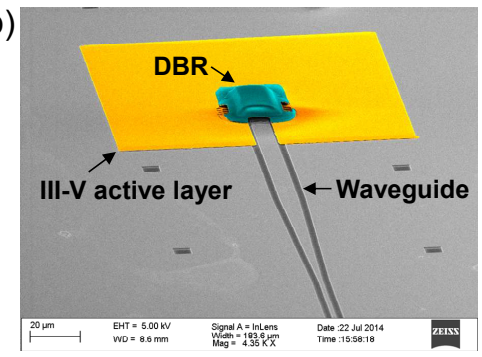

Fig. 1. (a) Schematic of the hVCL. (b) Scanning electron microscope image of a fabricated hVCL.

\section{Device structure, Fabrication, and Characterization}

As shown in Fig. 1(a), the hVCL consists of a dielectric distributed Bragg reflector (DBR), a HCG reflector, and a III-V active layer which is surrounded by two vertically aligned reflectors. A Si waveguide is placed near the one end of the HCG region so that waveguide modes can be excited by HCG modes, leading to the in-plane emission of light into the Si waveguide [1]. The hVCL was fabricated by using CMOS-compatible processes. First, the HCG and waveguide patterns were formed on a silicon-on-insulator (SOI) wafer by using electron-beam lithography and dryetching process. The HCG was designed as a TM-polarization mirror operating around $1550 \mathrm{~nm}$. Then, the III-V active layer including $7 \mathrm{InGaAlAs} / \mathrm{InGaAlAs}$ quantum wells (QWs) was directly bonded to the SOI wafer at $300^{\circ} \mathrm{C}$ and the InP substrate was removed. For the top mirror, 6 pairs of $\mathrm{Si} / \mathrm{SiO}_{2}$ DBR layers were deposited. Lastly, wet sacrificial etching was conducted to introduce an air gap between the active layer and the HCG. The fabricated hVCL sample was 

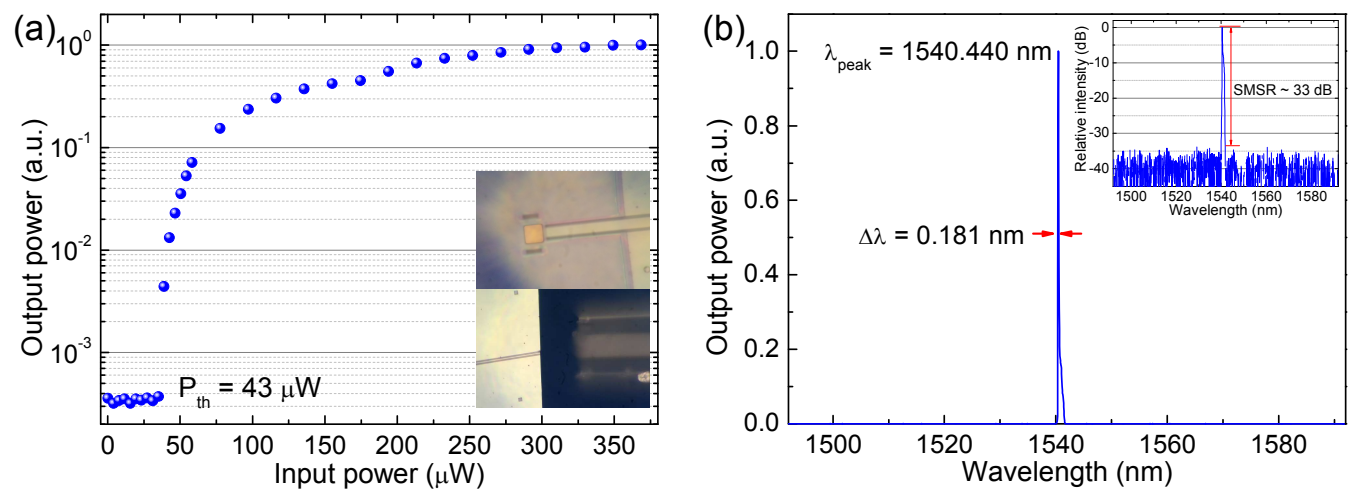

Fig. 2. (a) Measured light-out versus light-in graph. Inset: microscope image of a fabricated laser (upper) and the end facet of the Si waveguide and the multi-mode fiber (lower). (b) Lasing spectrum at an input power of $\sim 370 \mu \mathrm{W}$. Inset: the spectrum in $\mathrm{dB}$ scale.

cleaved through the Si waveguide for measuring the light coupled to the Si waveguide. One of the fabricated samples is shown in Fig. 1(b). The hVCL was characterized at room temperature under 980-nm pulsed pumping with a 20 -ns wide pulses at $5-\mathrm{MHz}$ repetition rate to avoid heating. The pump light was focused on the sample using a $\times 50$ nearinfrared objective lens. The in-plane output light from the $1.35-\mathrm{mm}$ long waveguide was collected by a multi-mode fiber, as shown in the lower inset of Fig. 2(a), which is connected to an optical spectrum analyzer (OSA) (Yokogawa AQ637D). The resolution for the OSA was set to $0.2 \mathrm{~nm}$.

\section{Result and Discussion}

The light-in light-out (LL) characteristic of the hVCL is presented in Fig. 2(a). The input power is the absorbed power in the QW and barrier layers, which is estimated considering the loss through the alignment optics, the DBR transmittance of $\sim 62.2 \%$ at $980 \mathrm{~nm}$, and the QW and barrier absorption efficiency of $30 \%$. Lasing clearly starts at threshold input power, $\mathrm{P}_{\text {th }}$ of $\sim 43 \mu \mathrm{W}$. In the continuous wave case, this value corresponds to a threshold current, $I_{\text {th }}$ of $\sim 0.34 \mathrm{~mA}\left(=\mathrm{P}_{\mathrm{th}} /(\right.$ photon energy at $980 \mathrm{~nm}) \times($ electron charge $) /($ duty cycle of the pulsed input $\left.)\right)$. It is noteworthy that this threshold current is considerably lower than commercially-available 1550-nm single-mode VCSELs, e.g. 1 $\mathrm{mA}$ to $2 \mathrm{~mA}$ [2]. Therefore, this hVCL structure has the potential to operate at threshold currents comparable to the best VCSELs, even when taking into account an expected increase in threshold for an electrically-injected structure. As shown in Figure 2(b), the in-plane output has a peak wavelength of $1540 \mathrm{~nm}$ and a full-width at half-maximum linewidth of $0.18 \mathrm{~nm}$ which is limited by the spectrum analyser resolution. As shown in the inset in Fig. 2(b), the side-mode suppression ratio (SMSR) is $\sim 33 \mathrm{~dB}$.

\section{Summary}

We have experimentally demonstrated an optically-pumped hybrid vertical-cavity laser emitting into an in-plane Si waveguide, with low threshold and high SMSR. We believe that the hVCL is a promising high-performance low-cost light source for chip-level optical interconnects.

\section{Acknowledgement}

The authors gratefully acknowledge supports by the Danish Research Council through the FTP project (Grant No. 11-106620) as well as the Villum Fonden via the NATEC Centre of Excellence.

\section{References}

1. I.-S. Chung et al., "Silicon-photonics light source realized by III-V/Si-grating-mirror laser," Appl. Phys. Lett. 97, 151113 (2010).

2. For example, www.raycan.com, www.vertilas.com 